Check for updates

Cite this: RSC Adv., 2017, 7, 41896

Received 5th August 2017

Accepted 15th August 2017

DOI: $10.1039 / c 7 r a 08659 h$

rsc.li/rsc-advances

\section{Diameter-controlled Cu nanoparticles on saponite and preparation of film by using spontaneous phase separation $\uparrow$}

\author{
Masaya Miyagawa, ${ }^{a}$ Akane Shibusawa, ${ }^{a}$ Kaho Maeda, ${ }^{a}$ Akiyoshi Tashiro, ${ }^{b}$ \\ Toshiki Sugaib and Hideki Tanaka*a
}

\begin{abstract}
Cu nanoparticles have attracted much attention due to their optical, catalytic, and electrical properties. Their syntheses, however, have required hazardous reducing agents. In addition, they are easily oxidized, which destroys their properties. It has also not been possible to control the diameter and the dispersibility for further applications in functional materials. To solve these problems, we have recently synthesized oxide-free $\mathrm{Cu}$ nanoparticles adsorbed on kaolinite, a non-dispersive layered clay, by environmentally friendly photoreduction. Focusing on the layer charge of the clay, we report diameterand dispersibility-controlled $\mathrm{Cu}$ nanoparticles on saponite, a dispersive clay, in the present study. The obtained oxide-free $\mathrm{Cu}$ nanoparticles were stable under $\mathrm{N}_{2}$ atmosphere, while they exhibited a fast back reaction to $\mathrm{Cu}^{2+}$ upon exposure to fresh air. The diameter of the $\mathrm{Cu}$ nanoparticles could be controlled simply with $\left[\mathrm{Cu}^{2+}\right]$, because $\mathrm{Cu}^{2+}$ was adsorbed on saponite before the photoreduction. The dispersibility of the $\mathrm{Cu}$ nanoparticles was controlled without changing the diameter using the size of saponite aggregates: these were stably dispersed at low $\left[\mathrm{Cu}^{2+}\right]$, and flocculated at high $\left[\mathrm{Cu}^{2+}\right]$. The flocculates could be collected by filtration and the obtained viscous paste was useful for preparing a film with the optical properties of the $\mathrm{Cu}$ nanoparticles without aggregation. Therefore, the present study revealed that combining the adsorption of $\mathrm{Cu}^{2+}$ on saponite with photoreduction is a novel and environmentally friendly synthetic method for controlling the diameter and the dispersibility of $\mathrm{Cu}$ nanoparticles, and it has the potential to further their applications in functional materials.
\end{abstract}

\section{Introduction}

As an alternative to precious metals such as $\mathrm{Pt}, \mathrm{Au}$, and $\mathrm{Rh}, \mathrm{Cu}$ nanoparticles (NPs) have attracted much attention due to their large reserves. ${ }^{\mathbf{1 - 4}}$ Their optical, catalytic, and electrical properties have recently been energetically investigated. ${ }^{3-11}$ However, the $\mathrm{Cu}$ NPs have generally been synthesized by a chemical reduction method, which requires hazardous reducing agents such as sodium borohydride ${ }^{3,5,8}$ and hydrazine. ${ }^{2}$ While reduction with a safer agent has also been investigated, ${ }^{\mathbf{9}, \mathbf{1 2}}$ extensive efforts have been required to optimize multiple conditions such as reaction temperature and reducing agent concentration. In contrast, photoreduction has shown potential since the 21st century due to its environmentally friendly conditions. ${ }^{\mathbf{1 3 - 1 6}}$

\footnotetext{
${ }^{a}$ Department of Applied Chemistry, Faculty of Science and Engineering, Chuo University, 1-13-27, Kasuga, Bunkyo-ku, Tokyo, Japan. E-mail: htanaka@kc.chuo-u. $a c . j p$

${ }^{b}$ Department of Chemistry, Faculty of Science, Toho University, 2-2-1, Miyama, Funabashi-shi, Chiba, Japan

$\dagger$ Electronic supplementary information (ESI) available. See DOI: $10.1039 / \mathrm{c} 7 \mathrm{ra} 08659 \mathrm{~h}$
}

In most of the previous studies, the metal NPs have been stabilized by protection with polymers ${ }^{\mathbf{1 4 , 1 7}}$ or surface modifiers such as carboxylates ${ }^{18,19}$ and thiolates. ${ }^{20,21}$ In contrast, we have previously reported protectant-free $\mathrm{Cu}$ NPs adsorbed on kaolinite, a non-exfoliating layered clay mineral, by the photoreduction method. ${ }^{22}$ In this synthetic method, ethanol has been used as a sacrificial reagent instead of hazardous reducing agents, and the obtained NPs have been composed of pure $\mathrm{Cu}$ without oxides, which was notable because $\mathrm{Cu}$ NPs are generally easily oxidized to $\mathrm{Cu}_{2} \mathrm{O}$ and $\mathrm{CuO}^{2,23-25}$ It is true that oxide-free $\mathrm{Cu}$ NPs have been reported previously, in which the $\mathrm{Cu}$ NPs are protected with $\mathrm{SiO}_{2}$ (ref. 26) or oleic acid, ${ }^{27}$ but it has been reported that the protection decreases or destroys the reactivity of the NPs. ${ }^{28,29}$ In addition, multi-step procedures including heat treatment and centrifugation have also been indispensable in removing excess protectants and hazardous reducing agents, which lowers the yield of the $\mathrm{Cu}$ NPs. From these viewpoints, our photoreduction method in combination with clay is promising because the protectant-free pure $\mathrm{Cu}$ NPs are obtained without further procedures by using a mild sacrificial reagent.

Unlike kaolinite with non-swelling properties and hence poor dispersibility, smectite clay minerals consist of negatively charged ultra-thin layers and interlayer cations for charge 
compensation. Because they swell in water, they are easily exfoliated, and the layers, so called nanosheets, are obtained as colloidal solutions. Despite numerous studies on layered clay minerals involving pillaring ${ }^{30,31}$ and hybridization with cationic molecules including dyes and metal complexes, ${ }^{32-34}$ however, there have been few studies on hybridization with metal NPs, and these have mainly been limited to Au and Ag NPs synthesized by chemical reduction. ${ }^{35-37}$ In addition, their diameter control on clay has not been achieved, even though the diameter strongly affects the reactivity of the Cu NPs. The diameter of metal NPs can be affected by various parameters, for instance reaction temperature, ${ }^{38}$ reaction time, ${ }^{39}$ metal salts, ${ }^{40}$ the ratio of ligand to metal salt, ${ }^{\mathbf{4 1}}$ and the presence of templates such as dendrimers. ${ }^{42}$ However, no significant change in the diameter has been achieved simply by changing $\left[\mathrm{Cu}^{2+}\right]$ on an adsorbent, for example graphene oxide. ${ }^{9}$ This problem may originate from uncontrollable particle growth in solutions without protectants. Hence, we have assumed that growth on an adsorbent would be key to controlling the diameter of the Cu NPs. In this regard, the negatively charged nanosheets of the smectite clay appear to be a promising adsorbent for $\mathrm{Cu}$ NPs, because they can effectively adsorb metal cations. ${ }^{\mathbf{4 3 , 4 4}}$ In this case, nucleation and growth should occur on the adsorbent and not in the solution. In other words, the growth of the reduced $\mathrm{Cu}$ seeds is expected to be suppressed on clay, resulting in diameter control of the Cu NPs with $\left[\mathrm{Cu}^{2+}\right]$. This strategy could not have been implemented in the previous study using kaolinite ${ }^{22}$ because this does not adsorb $\mathrm{Cu}^{2+}$, and particle growth of the Cu NPs has occurred in the solution before adsorption, resulting in a relatively large diameter $(80 \mathrm{~nm})$.

As well as diameter control, dispersibility control of colloidal NPs has also been important in their extraction, selective dispersion, and recycling. ${ }^{\mathbf{4 5 - 4 8}}$ However, contrary to the dispersibility of the polymer-grafted NPs, that of the adsorbed NPs has scarcely been investigated, even though they may become a promising material due to their high reactivity originating from the absence of protectants. In this regard, hybridization of the $\mathrm{Cu}$ NPs with clay can be a first experimental example because the dispersibility of the Cu NPs should be explained by that of the adsorbent, clay nanosheets. In this case, the Cu NPs can be collected as clay-hybridized flocculates and be further applied to NP films. Furthermore, because the $\mathrm{Cu}$ NPs are expected to be separated from each other by the clay nanosheets, the film can be obtained without aggregation of the $\mathrm{Cu}$ NPs, which has potential for optical materials.

Even though combination of the environmentally friendly photoreduction method and the layered clay minerals has not yet been studied, it will provide novel viewpoints with respect to both protectant-free metal NPs and inorganic layered materials. In the present study, we have synthesized highly reactive oxidefree $\mathrm{Cu}$ NPs on saponite (Sapo) by the photoreduction method. We have controlled the diameter and the dispersibility of the $\mathrm{Cu}$ NPs by changing $\left[\mathrm{Cu}^{2+}\right]$ and [Sapo]. By using flocculation and following conventional filtration, we have developed a novel preparation method for a composite film of Cu NPs, which were not aggregated due to isolation by the clay matrix.

\section{Experimental}

Copper nanoparticles were synthesized by a photoreduction method similar to that reported previously. ${ }^{22}$ Copper(II) acetate monohydrate (purity $>99.0 \%$ ) and ethanol (purity $>99.5 \%$ ) were purchased from Wako Pure Chemical Industries. Synthetic saponite was purchased from Kunimine Ind. Co., and its elemental composition and cation exchange capacity (CEC) were determined to be $\left(\mathrm{Na}_{0.25} \mathrm{Mg}_{0.07}\right)\left(\mathrm{Mg}_{2.98} \mathrm{Al}_{0.01}\right)\left(\mathrm{Si}_{3.6} \mathrm{Al}_{0.4}\right)$ $\mathrm{O}_{10}(\mathrm{OH})_{2}$ and 80 meq. per $100 \mathrm{~g}$, respectively. ${ }^{49}$ Sapo was dispersed in Milli-Q water, and $5 \mathrm{~mL}$ of copper acetate aqueous solution and $1 \mathrm{~mL}$ of ethanol were added to the dispersion drop by drop with stirring. The total volume of the mixed solution was adjusted to $10 \mathrm{~mL}$ by adding water. The solution was irradiated with UV light from a $\mathrm{Hg}$ lamp (Hamamatsu Photonics, L9588-01A) for $2 \mathrm{~h}$ with stirring. To prepare the $\mathrm{Cu}$ NP film, a small amount of the Cu NP paste obtained by filtration of flocculates was cast on a glass plate, sandwiched with another glass plate, and dried under $\mathrm{N}_{2}$ atmosphere.

UV-vis-NIR spectra were measured with a UV-vis-NIR spectrometer (Shimadzu, UV-3600) using a rectangular quartz cuvette having an optical length of $10 \mathrm{~mm}$. The photoirradiated solution was dropped on a glass plate under $\mathrm{N}_{2}$ atmosphere and dried in vacuum. X-ray diffraction (XRD) measurement of the obtained powder sample was performed with an X-ray diffractometer (Rigaku, Smartlab) with $\mathrm{CuK} \alpha$ radiation $(0.154 \mathrm{~nm})$. For transmission electron microscopy (TEM) measurement, the photoirradiated solution was diluted to avoid charge-up originating from the insulative saponite and dropped on a collodioncoated copper grid under $\mathrm{N}_{2}$ atmosphere. TEM images of the dried grid were observed with a transmission electron microscope (FEI Company, Tecnai G2 F20 S-TWIN), operated at $120 \mathrm{kV}$, and with a field-emission scanning electron microscope (Hitachi High-Technologies, S-5500) operated at $25 \mathrm{kV}$. Dynamic light scattering (DLS) measurement of the photoirradiated solution was performed with a particle size analyser (Otsuka Electronics Co., Ltd., ELSZ-2000ZS).

\section{Results and discussion}

\section{Oxide-free Cu NPs adsorbed on Sapo}

When the mixed solution containing $0.5 \mathrm{mmol} \mathrm{L}^{-1}$ of $\mathrm{Cu}^{2+}$ and $0.5 \mathrm{~g} \mathrm{~L}^{-1}$ of Sapo was irradiated with UV light for $2 \mathrm{~h}$, a bright red solution was obtained, as shown in Fig. 1(a). This color was characteristic of surface plasmon resonance (SPR) of Cu NPs, ${ }^{50}$ suggesting that $\mathrm{Cu}^{2+}$ was reduced to $\mathrm{Cu}^{0}$ by UV irradiation. The photoirradiated solution (we call this sample $\{0.5: 0.5\}$ of $\mathrm{Cu}-$ Sapo, hereafter) was not separated into two phases but was stably dispersed. No large flocculates were observed with the naked eye, and the solution appeared homogeneous.

Fig. 1(b) shows a UV-vis-NIR extinction spectrum after UV irradiation. A band due to SPR was observed around $560 \mathrm{~nm}$, which originated from spherical Cu NPs. ${ }^{5,51}$ We note that the band of $\mathrm{Cu}^{2+}$ appearing around $780 \mathrm{~nm}$ before UV irradiation, which is shown in Fig. S1(a), $\dagger$ completely disappeared. Also, the spectrum before UV irradiation was almost consistent with the sum of the spectra of the pure copper acetate solution and the Sapo 
(a)

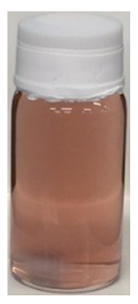

(c)

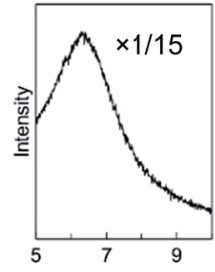

(b)
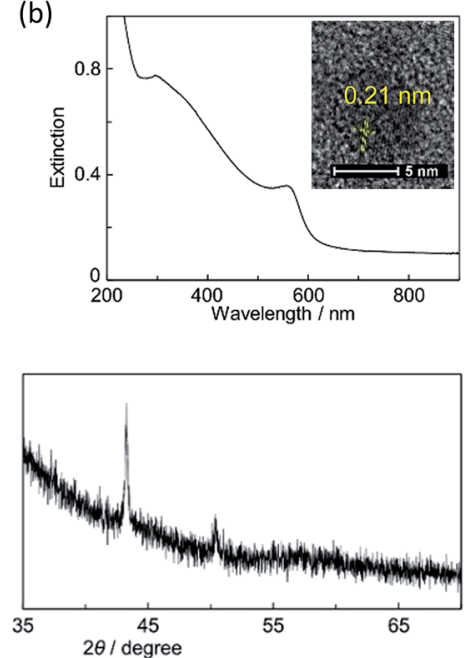

Fig. 1 (a) A photograph of the photoirradiated solution, (b) a UV-visNIR extinction spectrum and a high-resolution TEM image of the $\mathrm{Cu}$ NP (inset), and (c) an XRD pattern of $\{0.5: 0.5\}$.

dispersions shown in Fig. S1(b and c $\dagger$ ) respectively, suggesting that adsorption on Sapo did not induce a drastic change in the electronic state of $\mathrm{Cu}^{2+}$. Fig. 2(a) and $\mathrm{S} 2 \uparrow$ show TEM images of the dried sample at high and low magnifications, respectively. The spherical $\mathrm{Cu}$ NPs were observed without aggregation. From the histogram shown in Fig. S3(a), $\dagger$ the average diameter was determined to be $13 \pm 2 \mathrm{~nm}$, where the uncertainty represents one standard deviation. It should be noted that the blurring of the $\mathrm{Cu}$ NPs originates from the adsorbent, Sapo, which is insulative to induce charge-up. Similar results have also been reported in previous studies on clay-hybridized metal NPs..$^{52,53}$

(a)

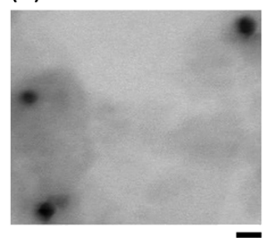

(d)

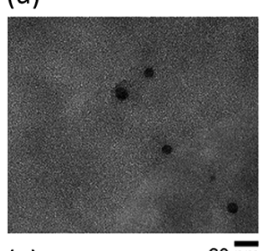

(g) (b)

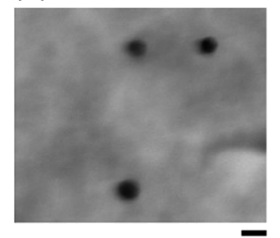

(e)

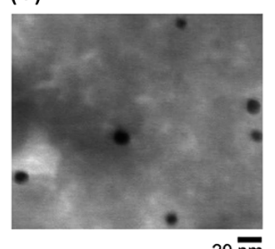

$20 \overline{\mathrm{nm}}$ (c)

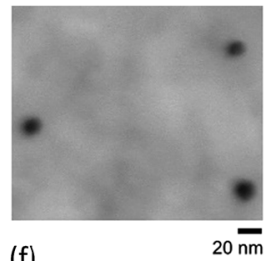

(f)

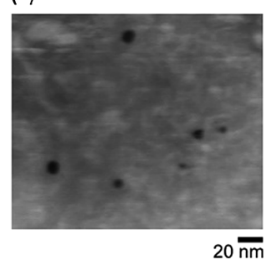

$20 \overline{\mathrm{nm}}$

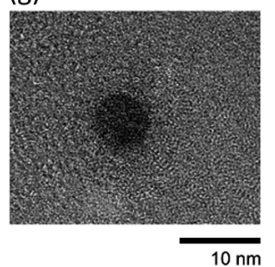

Fig. 2 TEM images of (a) $\{0.5: 0.5\}$, (b) $\{1: 1\}$, (c) $\{2: 2\}$, (d) $\{0.2: 0.5\}$, (e) $\{0.4: 1\}$, (f) $\{0.8: 2\}$, and (g) $\{0.1: 0.5\}$.

By comparison with a control experiment, where large $\mathrm{Cu}$ particles with sub-mm sizes were obtained in the absence of Sapo, as reported previously, ${ }^{22}$ it was found that the Cu NPs were adsorbed on Sapo and their particle growth was suppressed. In addition, while the photoirradiated solution was stable under $\mathrm{N}_{2}$ atmosphere, the solution turned pale light blue from bright red on exposure to fresh air, and copper acetate was detected with UV-vis-NIR spectroscopy, as in our previous studies. ${ }^{4,22,50} \mathrm{It}$ is noted that the bright red color faded away in 10 seconds, which was much faster than the color change in the Cu NPs protected by polyvinylpyrrolidone $(20 \mathrm{~min}) .{ }^{50} \mathrm{We}$ assume that this rapid decomposition of the Cu NPs on Sapo can be ascribed to the absence of protectants. Therefore, it was found that spherical Cu NPs were obtained on Sapo by UV irradiation and that their decomposition occurred quickly, which was probably due to the use of adsorbent rather than protectant.

A high-resolution TEM image is shown in the inset of Fig. 1(b). Crystal planes were observed with a fringe spacing of $0.21 \mathrm{~nm}$, which corresponded to the (111) plane of the fcc phase of metal copper according to JCPDS file no. 4-0836. Thus, it was found that the obtained NPs were composed of pure $\mathrm{Cu}$. This result contrasts with our previous study, in which a core-shell structure composed of $\mathrm{Cu}$-core and $\mathrm{Cu}_{2} \mathrm{O}$-shell was obtained when carbon nanotubes were used as the adsorbent. ${ }^{23}$ In other words, it was found that protectant-free $\mathrm{Cu}$ NPs were synthesized without oxides when Sapo was used as the adsorbent.

The absence of $\mathrm{Cu}_{2} \mathrm{O}$ and $\mathrm{CuO}$ was further confirmed by XRD measurement, as shown in Fig. 1(c). Two peaks were observed at $43.3^{\circ}$ and $50.4^{\circ}$, corresponding to the (111) and (200) planes of crystalline $\mathrm{Cu}$ metal, respectively. No peaks for $\mathrm{Cu}_{2} \mathrm{O}$ and $\mathrm{CuO}$ were observed at $36.4^{\circ}$ and $35.5^{\circ}$, respectively (JCPDS files no. 50067 and 48-1548). Thus, it was found that $\mathrm{Cu}^{2+}$ was reduced completely to $\mathrm{Cu}^{0}$ by UV irradiation. In addition, while the peak of the (001) plane of Sapo was observed at $6.4^{\circ}$, other strong peaks such as those at $53.2^{\circ}$ and $60.7^{\circ}$, which were assigned to the (150) and (310) planes, and the (060) and (330) planes, respectively, were not observed,$^{54}$ indicating that the layer structure of Sapo was destroyed. Because these peaks were also absent in the XRD pattern before UV irradiation, it was suggested that Sapo aggregated randomly when the copper acetate aqueous solution was added to the Sapo dispersion. The formation of the Sapo aggregates was further supported by DLS measurement. The average particle size after UV irradiation was found to be $560 \mathrm{~nm}$, which was much larger than that of the single nanosheet, $33 \mathrm{~nm},{ }^{55}$ indicating that Sapo aggregated microscopically in the solution rather than during the drying process for XRD sample preparation. Therefore, we concluded that Sapo supported the production of oxide- and protectantfree $\mathrm{Cu}$ NPs and also aggregated microscopically. The aggregation of Sapo is plausible because excess $\mathrm{Cu}^{2+}$ was added, which will be described later in relation to the CEC of Sapo.

\section{Diameter control of Cu NPs}

As well as $\{0.5: 0.5\}, \mathrm{Cu}$-Sapo was synthesized with various $\left[\mathrm{Cu}^{2+}\right]$ and [Sapo], and the TEM images are shown in Fig. 2 . The corresponding histograms are shown in Fig. S3. $\dagger$ The average 
diameters of the $\mathrm{Cu}$ NPs are summarized in Table 1 . The diameters were $13 \pm 3 \mathrm{~nm}$ in both $\{1: 1\}$ and $\{2: 2\}$, which were the same as that in $\{0.5: 0.5\}$ within the error range. Similar results were obtained in $\{0.4: 1\}$ and $\{0.8: 2\}$, whose diameters were the same as that in $\{0.2: 0.5\}, 8 \pm 2 \mathrm{~nm}$. Hence, it was found that the diameters did not change significantly if the ratio of $\left[\mathrm{Cu}^{2+}\right]$ to [Sapo] was constant. In contrast, the diameters decreased from $13 \pm 2 \mathrm{~nm}$ in $\{0.5: 0.5\}$ to $8 \pm 2$ and $6 \pm 2 \mathrm{~nm}$ in $\{0.2: 0.5\}$ and $\{0.1: 0.5\}$, respectively, indicating that the diameter of the Cu NPs could be controlled simply with $\left[\mathrm{Cu}^{2+}\right]$. Thus, the adsorption of $\mathrm{Cu}^{2+}$ on Sapo before photoreduction was found to be effective in controlling the diameter of the $\mathrm{Cu}$ NPs. In all cases, the SPR bands of the Cu NPs were observed, as shown in Fig. S4. $\dagger$

Since the microscopic aggregation of Sapo was induced by the added $\mathrm{Cu}^{2+}$ spontaneously adsorbing on Sapo, we expected the diameter of the Cu NPs to be changed by the drip rate of the copper acetate aqueous solution during sample preparation. When the drip rate was changed from $0.21 \mathrm{~mL} \min ^{-1}$ to $1.4 \mathrm{~mL} \min ^{-1}$, the average diameter of the Cu NPs in $\{1: 1\}$ increased from $13 \pm 3 \mathrm{~nm}$ to $20 \pm 2 \mathrm{~nm}$. A similar result was also obtained in the case of $\{2: 2\}$, where the diameter increased from $13 \pm 3$ to $24 \pm 6 \mathrm{~nm}$ with a fast drip rate. TEM images and histograms of $\{1: 1\}$ and $\{2: 2\}$ with the fast drip rate are shown in Fig. S5(a and b) and S3(a and b), $\dagger$ respectively. The increase in the diameter of the $\mathrm{Cu}$ NPs with the fast drip rate implies two possibilities: one is that larger Sapo aggregates containing more $\mathrm{Cu}^{2+}$ were formed with the fast drip rate, facilitating particle growth when $\mathrm{Cu}^{2+}$ was reduced by UV irradiation. The other possibility is that non-adsorbing $\mathrm{Cu}^{2+}$ was confined to the Sapo aggregates with the fast drip rate.

The formation of the large Sapo aggregates in the former scenario originates from the assumption that Sapo nanosheets aggregate more intensively with the fast drip rate, which was confirmed by the DLS measurement. The size of the Sapo aggregates with the fast drip rate was twice as large as that with the slow drip rate, and more $\mathrm{Cu}^{2+}$ was contained in each Sapo aggregate to increase the diameter of the Cu NPs. In contrast, if the latter is true, the amount of free $\mathrm{Cu}^{2+}$ in the solution should decrease with the fast drip rate. To clarify this, the solution was centrifuged before UV irradiation. Fig. S6 $\uparrow$ shows bands of $\mathrm{Cu}^{2+}$ in the UV-visNIR absorption spectra of the supernatant in $\{2: 2\}$ prepared with slow and fast drip rates. Because no difference was found between the two spectra, the latter scenario was improbable, and the

Table 1 The average diameters of the Cu NPs synthesized with various $\left[\mathrm{Cu}^{2+}\right]$ and [Sapo]

\section{Sample}

$\{0.1: 0.1\}$

$\{0.2: 0.5\}$

$\{0.4: 1\}$

$\{0.8: 2\}$

$\{0.5: 0.5\}$

$\{1: 1\}$

$\{2: 2\}$
Diameter/nm former was more plausible. Therefore, we concluded that the diameter of the $\mathrm{Cu}$ NPs could be controlled not only with $\left[\mathrm{Cu}^{2+}\right]$ but also with the drip rate during sample preparation.

\section{Dispersibility control of Cu NPs by microscopic aggregation of Sapo}

Unlike $\{0.5: 0.5\}$ of $\mathrm{Cu}-\mathrm{Sapo},\{2: 2\}$ was separated into two phases after keeping the solution unstirred for 1 day. We then examined the effects of $\left[\mathrm{Cu}^{2+}\right]$ and [Sapo] on the dispersibility of $\mathrm{Cu}-\mathrm{Sapo}$, as shown in Fig. 3. It was found that $\mathrm{Cu}-\mathrm{Sapo}$ was stably dispersed regardless of $[$ Sapo $]$ at $\left[\mathrm{Cu}^{2+}\right]=1 \mathrm{mmol} \mathrm{L}^{-1}$, while it flocculated spontaneously at [Sapo] $=2 \mathrm{~g} \mathrm{~L}^{-1}$ when $\left[\mathrm{Cu}^{2+}\right]$ was relatively high. In $\{2: 2\},\{2: 3\}$, and $\{2: 4\}$, the upper layer was totally colorless and transparent, while the lower layer was red. As expected, the SPR band of the $\mathrm{Cu}$ NPs was not observed in the UV-vis-NIR absorption spectrum of the upper layer, as shown in Fig. S7. $\uparrow$ Thus, Cu-Sapo was found to be flocculated. It should be noted that the flocculates were not precipitates, because they were transiently stable when the phase-separated solution was stirred. In other words, the stirred solution could be handled as a stable dispersion, because the flocculation took more than $1 \mathrm{~h}$.

Since the average diameter of the Cu NPs in $\{2: 2\}$ was the same as that in $\{0.5: 0.5\}$, the dispersibility of the Cu NPs could be controlled without changing their average diameter. In addition, because large flocculates were observed with the naked eye, the Sapo aggregates were expected to be relatively large. Indeed, the DLS measurement revealed that the size of $\{2: 2\}$ was larger than $10 \mu \mathrm{m}$. We note that the size was much larger than that of $\{0.5: 0.5\}$ at $560 \mathrm{~nm}$. Therefore, we concluded that the dispersibility of the $\mathrm{Cu}$ NPs was controlled by the size of the Sapo aggregates without changing the average diameter of the $\mathrm{Cu}$ NPs. (a)

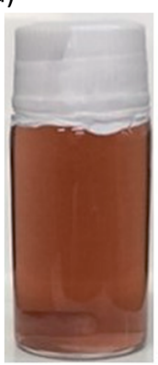

(e)

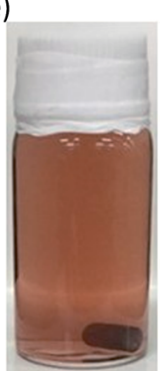

(b)

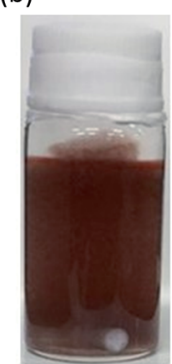

(f)

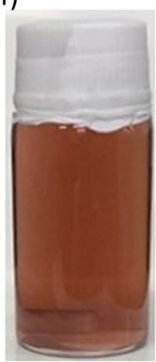

(c)

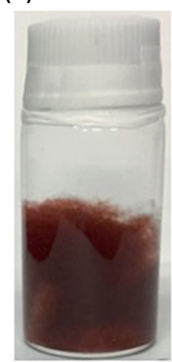

(g)

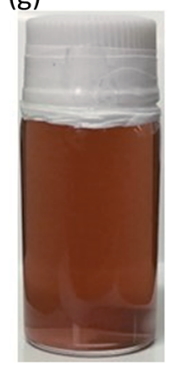

(d)

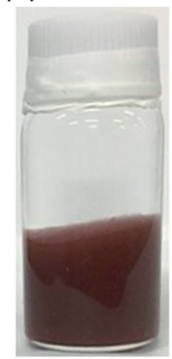

(h)

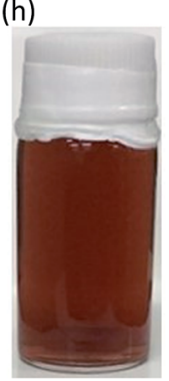

Fig. 3 Photographs of (a) $\{1: 2\}$, (b) $\{2: 2\}$, (c) $\{3: 2\}$, (d) $\{4: 2\}$, (e) $\{1: 1\}$, (f) $\{1: 2\}$, (g) $\{1: 4\}$, and (h) $\{1: 6\}$. 


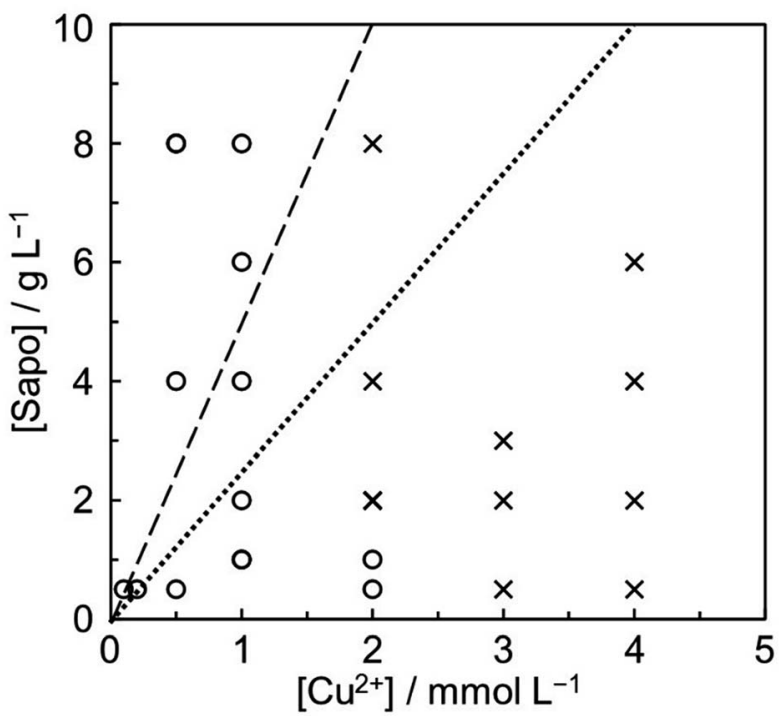

Fig. 4 Dispersion-state phase diagram of $\mathrm{Cu}$-Sapo. Symbols $\bigcirc$ and $\times$ represent 'dispersed' and 'flocculated', respectively, when the photoirradiated solution was kept unstirred for 1 day. The dotted and broken lines represent $100 \%$ and $50 \%$ of $\mathrm{Cu}^{2+}$ against the CEC of Sapo.

Fig. 4 shows a dispersion-state phase diagram of $\mathrm{Cu}-$ Sapo. At $\left[\mathrm{Cu}^{2+}\right] \leq 1 \mathrm{mmol} \mathrm{L}^{-1}, \mathrm{Cu}-$ Sapo was stably dispersed regardless of [Sapo]. In contrast, it was mostly flocculated at $\left[\mathrm{Cu}^{2+}\right]$ $\geq 2 \mathrm{mmol} \mathrm{L}^{-1}$. The fact that $\mathrm{Cu}$-Sapo was not flocculated regardless of [Sapo] at $\left[\mathrm{Cu}^{2+}\right] \leq 1 \mathrm{mmol} \mathrm{L}^{-1}$ seems rather peculiar, because the $\mathrm{Cu}$ NPs were dispersed in the presence of negatively charged Sapo nanosheets and cations for charge compensation, which should be regarded as a salt to induce the flocculation of general colloidal NPs. We assume that the adsorption on Sapo enabled the Cu NPs to be stably dispersed even at high [Sapo]. In other words, the aggregation of Sapo was induced by the $\mathrm{Cu}$ NPs. In addition, the DLS measurement revealed that the Sapo aggregates were relatively small at low $\left[\mathrm{Cu}^{2+}\right]$, suggesting that the extent of aggregation of Sapo was low, resulting in the stable dispersion of $\mathrm{Cu}$-Sapo. In contrast, larger Sapo aggregates were formed at high $\left[\mathrm{Cu}^{2+}\right]$, followed by the flocculation of $\mathrm{Cu}-\mathrm{Sapo}$, probably because the presence of more $\mathrm{Cu}$ NPs caused the Sapo nanosheets to aggregate enough to cause macroscopic flocculation. Also, because $\mathrm{Cu}-\mathrm{Sapo}$ was stably dispersed in $\{2: 0.5\}$ and $\{2: 1\}$ but flocculated in $\{2: 2\}$, it was suggested that the more nanosheets there were, the larger the aggregates became, resulting in macroscopic flocculation at high [Sapo]. Therefore, we concluded that the dispersibility of $\mathrm{Cu}$-Sapo was controlled predominantly by $\left[\mathrm{Cu}^{2+}\right]$ without changing the diameter of the Cu NPs, and the adsorption of the $\mathrm{Cu}$ NPs on Sapo enabled $\mathrm{Cu}-\mathrm{Sapo}$ to be stably dispersed even at high [Sapo] if $\left[\mathrm{Cu}^{2+}\right]$ was low.

\section{Preparation of $\mathrm{Cu}-\mathrm{Sapo}$ film from the flocculates}

To prepare the $\mathrm{Cu}-\mathrm{Sapo}$ film, the flocculates were filtered, as shown in Fig. S8(a). $\dagger$ Because the Cu NPs were obtained not as a precipitate but as transiently dispersed flocculates, filtration was needed to concentrate the $\mathrm{Cu}$-Sapo, contrary to the electroless deposition reported previously. ${ }^{56,57}$ The paste was so viscous that it did not drop even when it was turned upside down, as shown in Fig. 5(a). The collection rate of $\mathrm{Cu}-\mathrm{Sapo}$ by filtration was $88 \mathrm{wt} \%$, suggesting that the Cu NPs could be easily collected as flocculates. In contrast, the filtrate was colorless and transparent, as shown in Fig. S8(b). $\dagger$ Because no SPR band was observed in the UV-vis-NIR absorption spectrum of the filtrate shown in Fig. $\mathrm{S} 9, \dagger$ it was found that the $\mathrm{Cu}-\mathrm{Sapo}$ flocculates could be concentrated efficiently as a paste.

Fig. 5(b) shows a $\mathrm{Cu}$-Sapo film obtained from the paste in $\{2: 2\}$, whose preparation using the paste is described in the Experimental section. The film was bright red, like the flocculates, suggesting that the Cu NPs were not aggregated in the clay matrix. Indeed, no aggregation of Cu NPs with a similar diameter to those before filtration was observed in the TEM image prepared by casting a small amount of the paste on a grid followed by drying under $\mathrm{N}_{2}$ atmosphere, which is shown in Fig. 5(c). Fig. 5(d) shows a transmission spectrum of the central part of the film. Because no shoulder peak was observed on the long-wavelength side of the SPR band at $560 \mathrm{~nm}$, it was confirmed that the $\mathrm{Cu}$ NPs were not aggregated in the film. In addition, the SPR band was not shifted from that measured for the solution. This fact also confirms that non-aggregated $\mathrm{Cu}$ NPs with the same diameter are distributed in the film. All these facts should originate from the isolation of the $\mathrm{Cu}$ NPs with the clay matrix. Thus, it was found that the optical properties of the $\mathrm{Cu}$ NPs were preserved in the photoinactive clay film. Furthermore, the content of the Cu NPs in the film could be changed easily from $1.6 \mathrm{wt} \%$ in $\{2: 8\}$ to $34 \mathrm{wt} \%$ in $\{4: 0.5\}$. These values were calculated based on the facts that $\mathrm{Cu}^{2+}$ was completely reduced to $\mathrm{Cu}^{0}$, and that all the $\mathrm{Cu}$ NPs and Sapo aggregates were contained in the residue, both of which are supported by Fig. S7 and S9. $\uparrow$ Compared to general polymer-grafted NPs, the (a)

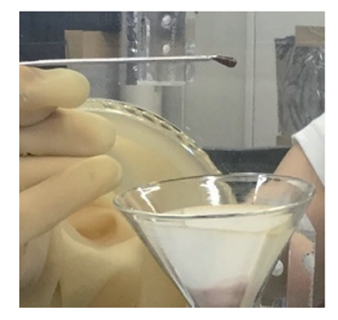

(c)

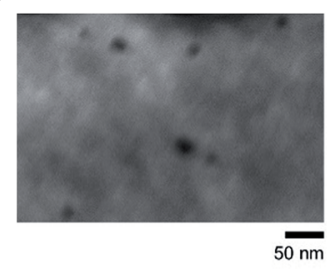

(b)

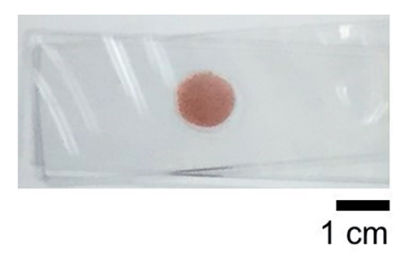

(d)

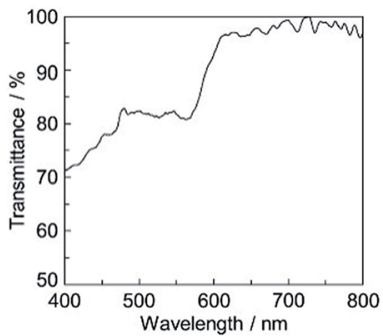

Fig. 5 Photographs of (a) the $\mathrm{Cu}$-Sapo paste turned upside down, prepared from the flocculates of $\{2: 2\}$ and (b) the film after drying, (c) a TEM image of the film, and (d) the visible transmission spectrum of the film. 
collection of $\mathrm{Cu}$-Sapo and the preparation of the film by flocculation appears to be a simple method because the dispersibility of $\mathrm{Cu}-$ Sapo was controlled easily by $\left[\mathrm{Cu}^{2+}\right]$.

It should be noted that flocculation played an important role in preparing the film because the paste obtained by filtration was viscous. For comparison, when the same experiment was performed with a stable dispersion, such as $\{1: 1\}$, the dripped dispersion was sandwiched over the whole glass, and the size of the film could not be controlled. In addition, since the dispersion could not be concentrated by filtration, the obtained film was almost transparent. In other words, the $\mathrm{Cu}$-Sapo film could not be prepared substantially. Thus, it was found that the spontaneous phase separation of the $\mathrm{Cu}$-Sapo solution was useful in preparing the film. In addition, the bright red film could be redispersed in water, because $\mathrm{Cu}$-Sapo existed as flocculates rather than precipitates. This characteristic is based on the hydrophilicity of Sapo. Therefore, we concluded that the spontaneous phase separation of $\mathrm{Cu}$-Sapo was useful in collecting the $\mathrm{Cu}$ NPs efficiently on a macroscopic scale as a paste or a film, where the $\mathrm{Cu}$ NPs were isolated in the clay matrix without aggregation. We also believe that filtration and the subsequent simple preparation method of the $\mathrm{Cu}$-Sapo film are useful both in restoring the Cu NPs and in applying them in optical and catalytic materials.

\section{Relationship between the dispersibility of $\mathrm{Cu}$-Sapo and CEC of Sapo}

One may wonder about the effect of the added amount of $\mathrm{Cu}^{2+}$ on the CEC of Sapo, which represents the maximum amount of cations that can be adsorbed. This was more than $100 \%$ in most of the $\mathrm{Cu}$-Sapo syntheses. For example, the added amount of $\mathrm{Cu}^{2+}$ was $250 \%$ of the CEC of Sapo in $\{0.5: 0.5\}$. In other words, $40 \%$ of the $\mathrm{Cu}^{2+}$ was adsorbed on Sapo, while the remaining $60 \%$ was dissolved in the solution. Indeed, when $\{0.5: 0.5\}$ was kept unstirred before UV irradiation, the solution separated into two phases, as shown in Fig. S10. $\uparrow$ Thus, it was found that large $\mathrm{Cu}^{2+}$-adsorbed Sapo aggregates were formed, resulting in flocculation. The DLS measurement further confirmed this finding, where the size of the Sapo aggregates before UV irradiation was larger than $10 \mu \mathrm{m}$. As well as the flocculates, the supernatant was also investigated. Fig. S11† shows a UV-vis-NIR absorption spectrum of the supernatant before UV irradiation. A weak band was observed around $800 \mathrm{~nm}$ with an intensity of 0.005 . Based on the facts that a band of $\mathrm{Cu}^{2+}$ was observed at $780 \mathrm{~nm}$ with an intensity of 0.010 for the pure copper acetate solution without Sapo, as shown in Fig. S1(a), $\dagger$ and $40 \%$ of the $\mathrm{Cu}^{2+}$ was adsorbed in the presence of Sapo, the obtained intensity was consistent with the estimated value. The bathochromic shift of the band is ascribed to a change in the molecular interaction of $\mathrm{Cu}^{2+}$ due to partial adsorption on Sapo.

Because of the excess amount of $\mathrm{Cu}^{2+}$ in $\{0.5: 0.5\}$, one may suspect that $\mathrm{Cu}$ nanocrystals are formed not only on Sapo but also in solution. In the latter case, secondary particles should be formed, which were not observed in the TEM image. In addition, because the size distribution of the Cu NPs was relatively monodisperse, it was suggested that photo-reduced $\mathrm{Cu}^{2+}$ was adsorbed on Sapo before overcoming the threshold for nucleation, which was consumed during the growth of the existing seeds produced from $\mathrm{Cu}^{2+}$ on Sapo by the initial UV irradiation. Furthermore, because Sapo becomes negatively charged again by the reduction of $\mathrm{Cu}^{2+}$ on Sapo, $\mathrm{Cu}^{2+}$ in the solution is assumed to be subsequently adsorbed on Sapo and should be consumed during the growth of the existing seeds on Sapo. The photoreduction mechanism of $\mathrm{Cu}^{2+}$ was discussed kinetically in detail in our previous study. ${ }^{22}$

\section{Conclusions}

In the present study, $\mathrm{Cu}$ nanoparticles were synthesized on saponite by an environmentally friendly photoreduction method. The obtained $\mathrm{Cu}$ nanoparticles contained no oxides and were stable under $\mathrm{N}_{2}$ atmosphere. The diameter could be easily controlled from 6 to $24 \mathrm{~nm}$ with $\left[\mathrm{Cu}^{2+}\right]$ and the drip rate of copper acetate solution. In addition, the dispersibility of the $\mathrm{Cu}$ nanoparticles was controlled mainly with $\left[\mathrm{Cu}^{2+}\right]$ without changing the diameter of the $\mathrm{Cu}$ nanoparticles, and the size of the saponite aggregates was found to play an important role. The $\mathrm{Cu}$ nanoparticles could be collected from the flocculates by filtration, and the film was prepared from the obtained paste, where the $\mathrm{Cu}$ nanoparticles were distributed in the clay matrix without aggregation. By combining the above results, we established a synthetic method for protectant-free pure $\mathrm{Cu}$ nanoparticles, where not only the diameter but also the dispersibility could be controlled, and which has the potential to further applications in functional materials.

\section{Conflicts of interest}

There are no conflicts to declare.

\section{Acknowledgements}

This work was supported by MEXT KAKENHI Grant Number 25248004 and by JSPS KAKENHI Grant Number 25410028. This work was also supported in part by the Supported Program for Strategic Research Foundation at Private Universities (20122016), Futaba Memorial Foundation, Shorai Foundation for Science and Technology, the Ogasawara Foundation for the Promotion of Science \& Engineering, and Foundation Advanced Technology Institute.

\section{Notes and references}

1 R. Reske, H. Mistry, F. Behafarid, B. R. Cuenya and P. Strasser, J. Am. Chem. Soc., 2014, 136, 6978.

2 D. Talukdar, G. Das, S. Thakur, N. Karak and A. Thakur, Catal. Commun., 2015, 59, 238.

3 B. J. Borah, S. J. Borah, L. Saikia and D. K. Dutta, Catal. Sci. Technol., 2014, 4, 1047.

4 M. Miyagawa, M. Yonemura and H. Tanaka, Chem. Phys. Lett., 2016, 665, 95.

5 I. Lisiecki, F. Billoudet and M. P. Pileni, J. Phys. Chem., 1996, 100, 4160. 
6 G. H. Chan, J. Zhao, E. M. Hicks, G. C. Schatz and R. P. Van Duyne, Nano Lett., 2007, 7, 1947.

7 S. Xu, B. Man, S. Jiang, J. Wang, J. Wei, S. Xu, H. Liu, S. Gao, H. Liu, Z. Li, H. Li and H. Qiu, ACS Appl. Mater. Interfaces, 2015, 7, 10977.

8 B. J. Borah, D. Dutta, P. P. Saikia, N. C. Barua and D. K. Dutta, Green Chem., 2011, 13, 3453.

9 I. Shown, H.-C. Hsu, Y.-C. Chang, C.-H. Lin, P. K. Roy, A. Ganguly, C.-H. Wang, J.-K. Chang, C.-I. Wu, L.-C. Chen and K.-H. Chen, Nano Lett., 2014, 14, 6097.

10 N. N. Jason, W. Shen and W. Cheng, ACS Appl. Mater. Interfaces, 2015, 7, 16760.

11 W.-H. Chung, H.-J. Hwang and H.-S. Kim, Thin Solid Films, 2015, 580, 61.

12 N. Kumar and L. S. B. Upadhyay, Appl. Surf. Sci., 2016, 385, 225.

13 R. Jin, Y. W. Cao, C. A. Mirkin, K. L. Kelly, G. C. Schatz and J. G. Zheng, Science, 2001, 294, 1901.

14 Y. Zhai, J. S. DuChene, Y.-C. Wang, J. Qiu, A. C. JohnstonPeck, B. You, W. Guo, B. DiCiaccio, K. Qian, E. W. Zhao, F. Ooi, D. Hu, D. Su, E. A. Stach, Z. Zhu and W. D. Wei, Nat. Mater., 2016, 15, 889.

15 H. Murayama, N. Hashimoto and H. Tanaka, Chem. Phys. Lett., 2009, 482, 291.

16 T. Nakato and T. Kasai, Mater. Lett., 2011, 65, 3402.

17 H. Murayama, N. Hashimoto and H. Tanaka, Chem. Phys. Lett., 2009, 482, 291.

18 M. Wu, D. N. Congreve, M. W. B. Wilson, J. Jean, N. Geva, M. Welborn, T. V. Voorhis, V. Bulović, M. G. Bawendi and M. A. Baldo, Nat. Photonics, 2016, 10, 31.

19 J. Deka, A. Paul and A. Chattopadhyay, Nanoscale, 2010, 2, 1405.

20 N. Nishida, Y. Kojima and H. Tanaka, Chem. Lett., 2014, 43, 1227.

21 N. R. Jana, L. Gearheart and C. J. Murphy, Langmuir, 2001, 17, 6782 .

22 M. Miyagawa, T. Maeda, R. Tokuda, A. Shibusawa, T. Aoki, K. Okumura and H. Tanaka, RSC Adv., 2016, 6, 104560.

23 N. Nishida, A. Miyashita, T. Tsukuda and H. Tanaka, Chem. Lett., 2013, 42, 168.

24 Y. Kobayashi and T. Sakuraba, Colloids Surf., A, 2008, 317, 756.

25 H.-J. Hwang, S.-J. Joo and H.-S. Kim, ACS Appl. Mater. Interfaces, 2015, 7, 25413.

26 S. Shiomi, M. Kawamori, S. Yagi and E. Matsubara, J. Colloid Interface Sci., 2015, 460, 47.

27 S. Jeong, S. H. Lee, Y. Jo, S. S. Lee, Y.-H. Seo, B. W. Ahn, G. Kim, G.-E. Jang, J.-U. Park, B.-H. Ryu and Y. Choi, J. Mater. Chem. C, 2013, 1, 2704.

28 C. Kim, B. L. Suh, H. Yun, J. Kim and H. Lee, ACS Catal., 2017, 7, 2294.

29 M. Cargnello, C. Chen, B. T. Diroll, V. V. T. Doan-Nguyen, R. J. Gorte and C. B. Murray, J. Am. Chem. Soc., 2015, 137, 6906.

30 R. Sasai, R. Watanabe and T. Yamada, Appl. Clay Sci., 2014, 93-94, 72.
31 S. Hamidouche, O. Bouras, F. Zermane, B. Cheknane, M. Houari, J. Debord, M. Harel, J.-C. Bollinger and M. Baudu, Chem. Eng. J., 2015, 279, 964.

32 D. Tokieda, T. Tsukamoto, Y. Ishida, H. Ichihara, T. Shimada and S. Takagi, J. Photochem. Photobiol., A, 2017, 339, 67.

33 H. Sato, K. Tamura, K. Ohara and S. Nagaoka, New J. Chem., 2014, 38, 132.

34 M. Colilla, M. Darder, P. Aranda and E. Ruiz-Hitzky, Chem. Mater., 2005, 17, 708.

35 L. Zhu, S. Letaief, Y. Liu, F. Gervais and C. Detellier, Appl. Clay Sci., 2009, 43, 439.

36 R. Zhang, M. Hummelgård and H. Olin, Langmuir, 2010, 26, 5823.

37 R. Patakfalvi, A. Oszkó and I. Dékány, Colloids Surf., A, 2003, 220, 45.

38 B. K. Park, S. Jeong, D. Kim, J. Moon, S. Lim and J. S. Kim, J. Colloid Interface Sci., 2007, 311, 417.

39 A. Umar, J. Lee, J. Dey and S.-M. Choi, Chem. Mater., 2016, 28, 4962.

40 A. Andrieux-Ledier, B. Tremblay and A. Courty, J. Phys. Chem. C, 2013, 117, 14850.

41 S. Chen and K. Kimura, Chem. Lett., 1999, 28, 1169.

42 M. Zhao, L. Sun and R. M. Crooks, J. Am. Chem. Soc., 1998, $120,4877$.

43 O. Abollino, M. Aceto, M. Malandrino, C. Sarzanini and E. Mentasti, Water Res., 2003, 37, 1619.

44 D. Malferrari, M. F. Brigatti, A. Laurora, L. Medici and S. Pini, J. Therm. Anal. Calorim., 2006, 86, 365.

45 S. Chen and K. Kimura, Langmuir, 1999, 15, 1075.

46 S. Srivastava, P. Agarwal and L. A. Archer, Langmuir, 2012, 28, 6276.

47 R. Mangal, P. Nath, M. Tikekar and L. A. Archer, Langmuir, 2016, 32, 10621.

48 R. Mangal, S. Srivastava and L. A. Archer, Nat. Commun., 2015, 6, 7198.

49 K. Okamoto, K. Tamura, M. Takahashi and A. Yamagishi, Colloids Surf., A, 2000, 169, 241.

50 N. Nishida, A. Miyashita, N. Hashimoto, H. Murayama and H. Tanaka, Eur. Phys. J. D, 2011, 63, 307.

51 I. Lisiecki and M. P. Pileni, J. Phys. Chem., 1995, 99, 5077.

52 K. K. K. Datta, M. Eswaramoorthy and C. N. R. Rao, J. Mater. Chem., 2007, 17, 613.

53 Á. Mastalir, G. Szöllösi, Z. Király and Z. Rázga, Appl. Clay Sci., 2002, 22, 9.

54 H. P. Cahoon, Am. Mineral., 1954, 39, 222.

55 R. Miyawaki, T. Sano, F. Ohashi, M. Suzuki, T. Kogure, T. Okumura, J. Kameda, T. Umezome, T. Sato, D. Chino, K. Hiroyama, H. Yamada, K. Tamura, K. Morimoto, S. Uehara and T. Yatta, J. Clay Sci. Soc. Jpn., 2010, 48, 158.

56 M. D. Susman, Y. Feldman, A. Vaskevich and I. Rubinstein, Chem. Mater., 2012, 24, 2501.

57 F. Muench, L. Sun, T. Kottakkat, M. Antoni, S. Schaefer, U. Kunz, L. Molina-Luna, M. Duerrschnabel, H.-J. Kleebe, S. Ayata, C. Roth and W. Ensinger, ACS Appl. Mater. Interfaces, 2017, 9, 771. 